\title{
Effect of types of proximal femoral fractures on physical function such as lower limb function and Activities of Daily Living
}

\author{
Daisuke BAI, PT, MSc ${ }^{1,2}$, Mitsunori TokUdA, PT, $\mathrm{PhD}^{1,3}$, Taiki IkEMoto, $\mathrm{PT}^{1}$, Shingo SugIMORI, $\mathrm{PT}^{1}$, \\ Shoki OKAMURA, $\mathrm{PT}^{1}$, Yuka YAMADA, $\mathrm{PT}^{1}$, Yuna TOMITA, $\mathrm{PT}^{1}$, \\ Yuki MORIKAWA, $\mathrm{PT}^{1}$ and Yasuhito TANAKA, MD, $\mathrm{PhD}^{2,4}$ \\ ${ }^{1)}$ Department of Rehabilitation, Heisei Memorial Hospital, Japan \\ ${ }^{2)}$ Graduate School of Medicine, Musculoskeletal Reconstructive Surgery, Nara Medical University, Japan \\ ${ }^{3)}$ Graduate School of Health Sciences, Kio University, Japan \\ ${ }^{4)}$ Department of Orthopedic Surgery, Nara Medical University, Japan
}

\begin{abstract}
Objectives: This study aimed to assess physical function such as lower limb function and Activities of Daily Living after surgery for proximal femoral fractures ( unstable medial femoral neck fracture and trochanteric fracture). Methods: This study enrolled 68 patients with proximal femoral fractures. Isometric knee extension strength (IKES), the Japanese Orthopedic Association (JOA) hip score, and the number of days required to develop straight leg raising, transfer, and T-caneassisted gait abilities to become independent were assessed. Patients were classified based on the types of proximal femoral fractures, namely unstable medial femoral neck fracture (bipolar hip arthroplasty [BHA] group), stable trochanteric fracture (S group), and unstable trochanteric fracture (US group). Results: IKES and the JOA hip score were significantly better in the BHA group than in the S and US groups. IKES and the JOA hip score were significantly worse in the US group than in the BHA and S groups. Both transfer and T-cane-assisted gait abilities of patients in the BHA and S groups were indifferent. However, all physical functions were significantly worse in the US group. Conclusions: Our study results suggested that physical therapists plan the different rehabilitation program for the patients with proximal femoral fractures who were classified into three types, namely unstable medial femoral neck fracture, stable trochanteric fracture, and unstable trochanteric fracture, instead of two types.

Key words: Proximal Femoral Fractures, Surgical Approach, Lower Limb Function, Activities of Daily Living (ADL), Physical Function
\end{abstract}

(Phys Ther Res 24: 24-28, 2021)

$\mathbf{P}_{\mathrm{r}}$ ing injuries among the elderly individuals. Loss of gait ability and quality of life after proximal femoral fracture are more severe among majority of the patients ${ }^{1-3)}$. Furthermore,

Received: May 12, 2020

Accepted: July 22, 2020

Advance Publication by J-STAGE: September 28, 2020

Correspondence to: Daisuke Bai, Department of Rehabilitation, Heisei Memorial Hospital/Graduate School of Medicine, Musculoskeletal Reconstructive Surgery, Nara Medical University, Japan, 827 Shijyocho, Kashihara, Nara 634-0813, Japan

\# e-mail: h1371019@kio.ac.jp

doi: 10.1298/ptr.E10050 the inability to walk after hospital discharge and presence of delirium are independent predictors of 1-year mortality. In addition, the possibility of not recovering gait ability at hospital discharge and 1-year mortality after proximal femoral fracture were higher among older patients who had severe cognitive impairment, lower functional level before injury, and those who experienced postoperative delirium and pressure ulcers ${ }^{3)}$. Therefore, early recovery of gait ability is crucial for the patients.

Proximal femoral fractures are divided into two fracture types: medial femoral neck fracture and trochanteric fracture. Researchers have previously reported that the clinical prognosis of medial femoral neck fracture was con- 
siderably better than that of trochanteric fracture ${ }^{4-7)}$. According to Evans classification, trochanteric fractures were divided into the stable and unstable types ${ }^{8)}$. The short-term clinical results of individuals with stable type were better than of those with unstable type for gait ability ${ }^{9)}$. Therefore, considering these reports, it is important to subdivide proximal femoral fractures into the three-fracture type. However, no studies have reported physical function such as lower limb function and Activities of Daily Living in the threefracture type of the proximal femoral fracture. We hypothesized that physical function such as lower limb function and Activities of Daily Living decrease in the following order: medial femoral neck fracture, stable type of trochanteric fracture, and unstable type of trochanteric fracture.

This study aimed to assess physical function such as lower limb function and Activities of Daily Living after surgery for proximal femoral fractures (unstable medial femoral neck fracture and trochanteric fracture).

\section{Material and Methods}

\section{Patients}

This study recruited 382 patients who experienced proximal femoral fractures from March 2016 to February 2019. The inclusion criteria were independent gait ability with or without T-cane-assistance before the fracture. And the period of hospitalization for the patients was for more than 4 weeks. The exclusion criteria were presence of dementia (Mini Mental State Examination score: <23) and a history of cerebrovascular or osteoarthritis disorder that caused gait disorder. In this study, the period of hospitalization for the patients with proximal femoral fractures who underwent intramedullary nail fixation (hanson pin or cannulated cancellous screw) was within two weeks. Therefore, they were excluded in this study. In total, 68 patients with proximal femoral fractures who underwent bipolar hip arthroplasty (BHA; BHA group) or intramedullary nail fixation (gamma locking nail; gamma group) met the inclusion criteria and were enrolled in this study. After surgery, all the patients were divided based on the surgery type. The BHA group comprised 34 patients and the gamma group comprised 34 patients. Moreover, the gamma group patients were divided into the stable type ( $\mathrm{S}$ group; 21 patients) and the unstable type (US group; 13 patients) ${ }^{8)}$. This study was approved by the institutional review board and conformed to the Declaration of Helsinki. All the procedures in this study were approved by the Ethics Committee of the Heisei Memorial Hospital (H15-1). No infection was observed in the hip during the entire postoperative course.

All the patients followed the same postoperative rehabilitation protocol and they did not drop out for this protocol. They were instructed to increase the range of hip motion starting the day after the surgery and to bear as much weight as they could tolerate starting the day after the treat- ment.

\section{Assessment of outcome}

Relevant descriptive characteristics such as age, height, weight, and body mass index (BMI) were extracted from the medical records of the patients. We evaluated isometric knee extension strength (IKES) and the Japanese Orthopedic Association (JOA) hip score at 1, 2, 3, and 4 weeks after the surgery and discharge.

IKES was measured using a hand-held dynamometer ( $\mu$-tas F-1, ANIMA Corp., Japan) with patients in a seated position and the knee flexed at a $90^{\circ}$ angle, as described previously ${ }^{10)}$. Patients were instructed to gradually increase the intensity of knee extension against the dynamometer for approximately 2 seconds, avoiding an explosive contraction, and to maintain their maximal force output for approximately 3 seconds. Two measurements were obtained and the maximum values were used for analysis. Furthermore, the laterality (affected side per non-affected side) was calculated.

Medical doctor, nurse, and physical therapist evaluated the number of days required to develop straight leg raising (SLR), transfer, and T-cane-assisted gait abilities to become independent. The independent criteria were more than 6 points on Functional Independence Measure.

\section{Statistical analyses}

IKES and the JOA hip score were analyzed using twoway analysis of variance (ANOVA) with repeated measurements to evaluate the fracture types and assessment time points (after 1, 2, 3, and 4 weeks of surgery and discharge).

The number of days required to develop SLR, transfer, and T-cane-assisted gait abilities by each group were compared using one-way ANOVA with repeated measures. Bonferroni corrections were applied to account for multiple comparisons. The significance level for all analyses was set at 5\%. SPSS version 18.0J for Windows (SPSS, Chicago, IL, USA) was used for analysis.

\section{Results}

Characteristics of patients of all the three groups are summarized in Table 1. No significant differences were noted in the mean age, height, weight, and BMI of patients of all the three groups.

The two-way ANOVA for both IKES and the JOA hip score showed a significant main effect of fracture types $(\mathrm{p}<$ $0.001)$, their interaction $(\mathrm{p}<0.01)$, and the assessment timepoint $(\mathrm{p}<0.001)$. According to Post hoc analyses, the BHA group had significantly better IKES (after 1, 2, 3, and 4 weeks of surgery) and JOA hip score (after 2, 3, and 4 weeks of surgery and discharge) compared with the $\mathrm{S}$ group. In addition, significantly better IKES (after 1, 2, 3, and 4 weeks of surgery and discharge) and JOA hip score 
Table 1. Patients' characteristics

\begin{tabular}{lrrrl}
\hline & $\begin{array}{c}\text { BHA } \\
\text { group }\end{array}$ & S group & US group & p value \\
\hline Age $($ year $)$ & $78.3 \pm 8.4$ & $81.2 \pm 6.1$ & $84.7 \pm 4.0$ & n.s. \\
Height $(\mathrm{cm})$ & $153.2 \pm 6.7$ & $152.2 \pm 8.2$ & $150.1 \pm 4.8$ & n.s. \\
Weight $(\mathrm{kg})$ & $59.1 \pm 9.3$ & $49.0 \pm 9.3$ & $45.5 \pm 9.2$ & n.s. \\
BMI $\left(\mathrm{kg} / \mathrm{m}^{2}\right)$ & $16.3 \pm 2.8$ & $16.0 \pm 2.4$ & $15.1 \pm 2.7$ & n.s. \\
\hline
\end{tabular}

Data expressed as means \pm standard deviations.

(after 1, 2, 3, and 4 weeks of surgery and discharge) were noted in the BHA group than in the US group ( $\mathrm{p}<0.001)$ according to the post hoc analyses. The IKES results (after $1,2,3$, and 4 weeks of surgery) of the $\mathrm{S}$ group were significantly better than those of the US group $(p<0.05)$. No differences were noted in the JOA hip scores of the $\mathrm{S}$ and US groups. The results are summarized in Table 2.

To become independent, the BHA, S, and US groups required $3.3 \pm 1.8,6.0 \pm 4.7$, and $17.1 \pm 6.2$ days, respectively, to develop SLR ability; $4.1 \pm 1.7,4.4 \pm 2.3$, and 8.0 \pm 4.7 days, respectively, to develop transfer ability; and $19.5 \pm 9.4,23.2 \pm 5.6$, and $38.6 \pm 10.8$ days, respectively, to develop T-cane-assisted gait ability. The BHA group required significantly less number of days to develop SLR ability than both the S and US groups. The BHA and S groups required the same number of days to develop both transfer and T-cane-assisted gait abilities. However, all physical functions were significantly worse in the US group. The results are summarized in Figure 1, 2, and 3.

\section{Discussion}

This study revealed the differences in physical function such as lower limb function and Activities of Daily Living after surgery by types of proximal femoral fractures, namely unstable medial femoral neck fracture and stable and unstable trochanteric fractures. Clinical prognosis was significantly worse in the US group. Therefore, considering the clinical prognosis, proximal femoral fractures should be subdivided into three types.

IKES was significantly higher after 1, 2, 3, and 4 weeks of surgery in the BHA group than in the S and US groups. However, at discharge, no differences were noted in IKES between the BHA and S groups. IKES was significantly worse in the US group at all assessment time points. Previously, researchers have reported that patients with trochanteric fracture experience a large of amount of bleeding $^{11,12)}$. Furthermore, patients in the US group have been reported to experience more bleeding than those in the $S$ group $^{13,14)}$. Thus, increased amount of bleeding resulted in swelling or edema in the thigh that in turn increased the internal pressure in the thigh, causing muscle weakness and limiting knee extension. In addition, the gamma locking nail invaded the vastus lateralis and knee extension is asso-

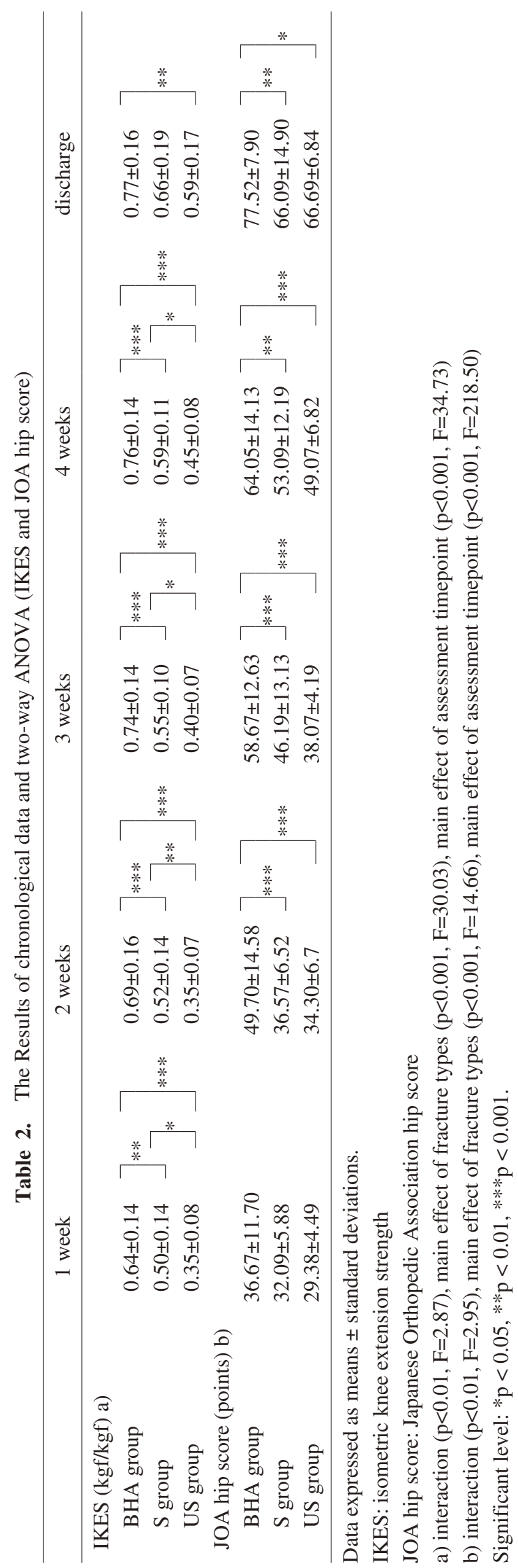




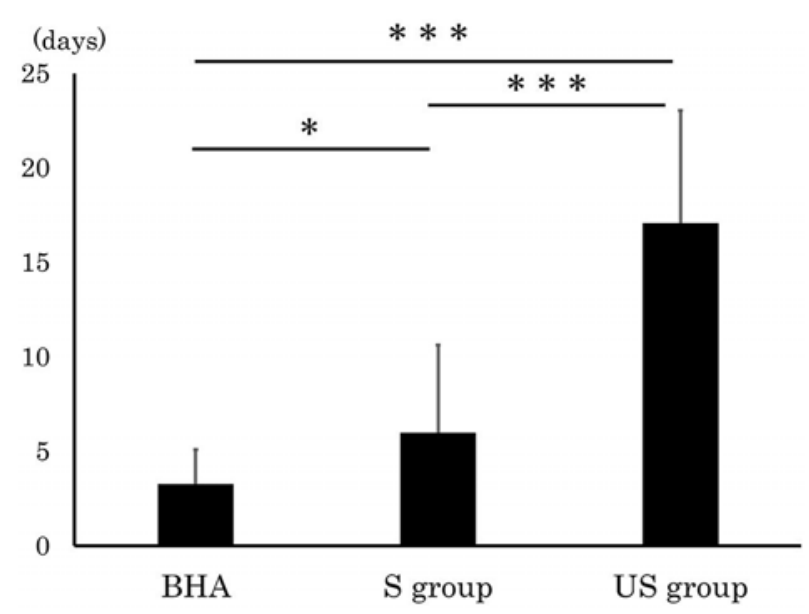

Figure 1. The number of days for SLR to become independent BHA group was significantly better than S group and US group. $\mathrm{S}$ group was significantly better than US group. *Significant level at $\mathrm{p}<0.05$. ***Significant level at $\mathrm{p}<0.001$.

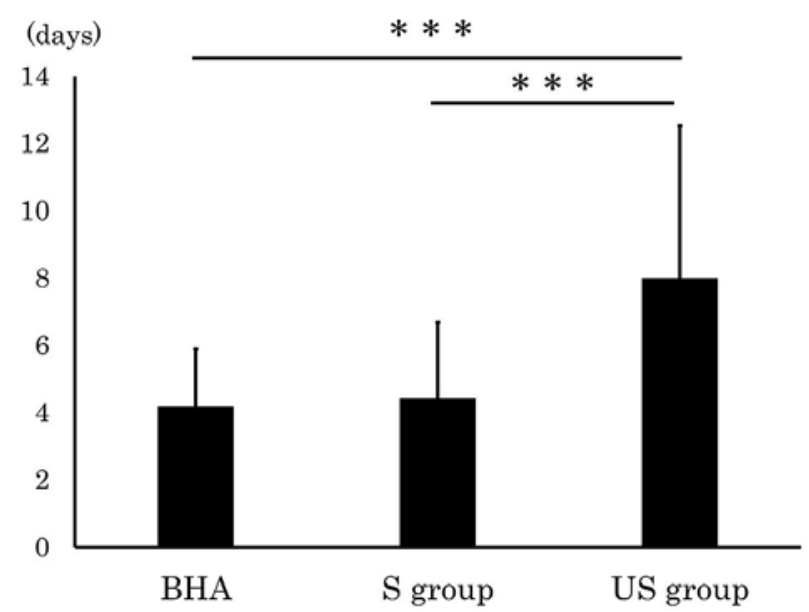

Figure 2. The number of days for transfer to become independent

US group was significantly worse than BHA and S group. There was no difference between BHA group and S group. ***Significant level at $\mathrm{p}<0.001$.

ciated with this muscle weakness. Hence, IKES was significantly higher and the number of days required to develop SLR ability to become independent were significantly lesser in the BHA group than in the S and US groups.

The JOA hip scores of the BHA group was higher than those of the $\mathrm{S}$ and US groups. There was no difference between $\mathrm{S}$ group and US group. The JOA hip score comprised pain, range of motion (ROM), gait ability, and activities of daily living (ADL) ability. Researchers have previously reported that the trochanteric fracture often caused periosteal pain, especially pain due to weight-bearing in the acute phase $\mathrm{e}^{15,16)}$. Few studies have reported about the postoperative passage for ROM after proximal femoral fractures. There was considerable amount of bleeding after the trochanteric fracture ${ }^{11,12}$. The swelling or edema with bleed-

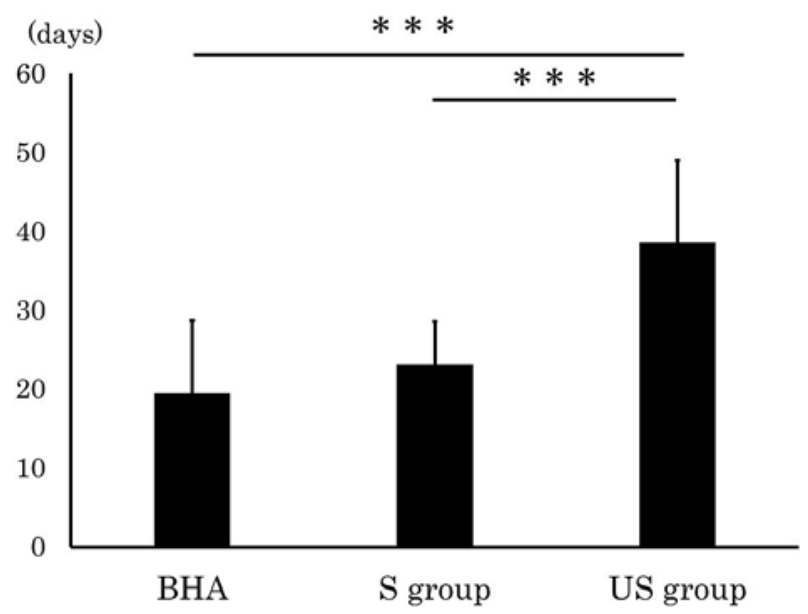

Figure 3. The number of days for T-cane gait to become independent

US group was significantly worse than BHA and S group. There was no difference between BHA group and $\mathrm{S}$ group. ***Significant level at $\mathrm{p}<0.001$.

ing is caused by the loss of soft tissue flexibility, resulting in lower ROM after trochanteric fracture. Considering ADL ability, the number of days required to develop SLR, transfer and gait abilities were significantly lesser in the BHA group than in the S and US groups. SLR ability is associated with getting out of bed. The earlier the patients get out of bed, the earlier they can become independent. In addition, SLR ability is associated with transfer ability. The patients of the US group experienced periosteal pain as well as weight-bearing pain. Therefore, due to pain and SLR inability, the US group required a long time to become independent as compared with the BHA and S groups. Furthermore, the transfer ability of US group was significantly worse than the BHA and S groups. And gait ability after proximal femoral fracture is associated with IKES ${ }^{18,19)}$. In addition, the decrease in hip flexion strength after lesser trochanter fracture affected the ability of swing initiation in poorly active patients and reduce their walking stability ${ }^{20)}$. Past researchers reported gait ability was most impaired in the US group ${ }^{9,17)}$. Therefore, the number of days to develop T-cane-assisted gait abilities to become independent was significantly worse in the US group than in the BHA and S groups. However, there was no differences between BHA and $\mathrm{S}$ groups. Therefore, we will assess to evaluate other muscle strength (for example, gluteus medius, gluteus maximus and so on) in the future. For all these reasons, the JOA hip scores of the BHA group was higher than those of the $\mathrm{S}$ and US group. However, there was no difference in the JOA hip scores of the $\mathrm{S}$ and US groups.

This study had some limitations. First, this study enrolled only $20 \%$ of all the patients with proximal femoral fracture because the level of inclusion criteria was high. Second, we did not evaluate the hip muscle strength. SLR ability is associated with iliopsoas muscle strength. The 
surgical invasion caused weakness in gluteus medius and maximus, the muscles associated with gait ability. In the future, we will change the inclusion criteria regarding dementia, recruit more patients and examine the other outcomes such as hip muscle strength. In addition, we intend to assess the relationship between the three types of fractures and physical function.

\section{Conclusion}

Our study results suggested that physical therapists plan the different rehabilitation program for the patients with proximal femoral fractures who were classified into three types, namely unstable medial femoral neck fracture, stable trochanteric fracture, and unstable trochanteric fracture, instead of two types.

Conflict of Interest: No benefits in any form have been received or will be received from a commercial party related directly or indirectly to the subject of this article. The authors received no financial support for the research, authorship, and/or publication of this article.

\section{References}

1) Sakamoto K, Nakamura T, et al.: Report of the Japanese Orthopaedic Association's 3-year project observing hip fractures at fixed point hospitals. J Orthop Sci. 2006; 11: 127-134.

2) Hagino $H$, Nakamura $T$, et al.: Sequential change in quality of life for patients with incident clinical fractures: prospective study. Osteoporos Int. 2009; 20: 695-702.

3) Dubjanin-Raspopovic E, Markovic Denic L, et al.: Use of early indicators in rehabilitation process to predict one-year mortality in elderly hip fracture patients. Hip Int. 2012; 22: 661-667.

4) Kristensen MT: Hip fracture-related pain strongly influences functional performance of patients with an intertrochanteric fracture upon discharge from the hospital. PM R. 2013; 5: 135-141.

5) Borgquist L, Nordell E, et al.: Outcome after hip fracture in different health care districts: rehabilitation of 837 consecutive patients in primary care 1986-88. Scand J Prim Health Care. 1991; 9: 244-251.

6) Tanaka R, Umehara T, et al.: Clinical prediction rule for declines in activities of daily living at 6 months after surgery for hip fracture repair. Arch Phys Med Rehabil. 2016; 97: 20762084.

7) Koval KJ, Skovron ML, et al.: Ambulatory ability after hip frac- ture. A prospective study in geriatric patients. Clin Orthop Relat Res. 1995; 310: 150-159.

8) Evans EM: The treatment of trochanteric fractures of the femur. J Bone Joint Surg Br. 1949; 31-B: 190-203.

9) Walheim G, Barrios C, et al.: Postoperative improvement of walking capacity in patients with trochanteric hip fracture: a prospective analysis 3 and 6 months after surgery. J Orthop Trauma. 1990; 4: 137-143.

10) Koblbauer IF, Lambrecht $Y$, et al.: Reliability of maximal isometric knee strength testing with modified hand-held dynamometry in patients awaiting total knee arthroplasty: useful in research and individual patient settings? a reliability study. BMC musculoskeletal disorders. 2011; 12: 249.

11) Wu JZ, Liu PC, et al.: A prospective study about the preoperative total blood loss in older people with hip fracture. Clin Interv Aging. 2016; 11: 1539-1543.

12) Smith GH, Tsang J, et al.: The hidden blood loss after hip fracture. Injury. 2011; 42: 133-135.

13) Ronga M, Bonzini D, et al.: Blood loss in trochanteric fractures: multivariate analysis comparing dynamic hip screw and Gamma nail. Injury. 2017; 48: 44-47.

14) Cuenca J, GarciaErce JA, et al.: Preoperative blood test results and type of fracture as transfusional risk factors in patients older than 65 years with trochanteric hip fracture. Rev Esp Anestesiol Reanim. 2004; 51: 515-522.

15) Mak JCS, Lattout I, et al.: Relationship between hip fracture subtypes, surgical procedure, and analgesia use. J Am Geriatr Soc. 2009; 57: 2378-2380.

16) Carvajal-Pedrosa C, Gomez-Sanchez RC, et al.: Comparison of outcomes of intertrochanteric fracture fixation using percutaneous compression plate between stable and unstable fractures in the elderly. J Orthop Trauma. 2016; 30: 201-206.

17) Brostrom LA, Barrios C, et al.: Clinical features and walking ability in the early postoperative period after treatment of trochanteric hip fractures: results with special reference to fracture type and surgical treatment. Ann Chir Gynaecol. 1992; 81: 6671.

18) Mangione K, Craik RL, et al.: Predictors of gait speed in patients after hip fracture. Physiother Can. 2008; 60: 10-18.

19) Palombaro KM, Craik RL, et al.: Determining meaningful changes in gait speed after hip fracture. Phys Ther. 2006; 86: 809-816.

20) Aprato A, Lo Baido R, et al.: Does lesser trochanter implication affect hip flexion strength in proximal femur fracture? Eur $\mathrm{J}$ Trauma Emerg Surg. 2015; 41: 523-529. 